\title{
Perioperative Beta-Blockers: Where do We Stand?
}

\author{
Li Shien Low, MD, Jennifer Heckman, MS III, Matthew DeCaro, MD
}

\section{Introduction}

Cardiovascular disease is a significant cause of morbidity and the leading cause of mortality in both the United States and worldwide. ${ }^{1,2}$ Given the considerable burden of disease, cardiac risk assessment is an especially important element of preoperative evaluation prior to noncardiac general surgery. ${ }^{3}$ Globally, an estimated 100 million adults undergo noncardiac surgery each year, ${ }^{4}$ more than one third of whom have underlying coronary artery disease (CAD).$^{5}$ Cardiac complications, including cardiac death, nonfatal myocardial infarction (MI), and nonfatal cardiac arrest, represent major causes of peri- and postoperative morbidity and mortality. ${ }^{6}$ The incidence of such cardiac complications is an estimated $1.4 \%$ in relatively unselected patients, ${ }^{7}$ with an even greater incidence $(2.4-5.8 \%)$ among those with or at risk of cardiac disease. $8,9,10,11,12,13$ Annually, more than one million patients are likely to experience such a complication. ${ }^{6}$ The magnitude of this problem can be measured not only in patient health outcomes and illness burden, but also in terms of the large cost subsequently imposed upon the healthcare system, especially as the number of noncardiac surgical patients at risk for adverse cardiac outcomes continues to increase. ${ }^{14}$

A common underlying pathophysiology posited to mediate perioperative myocardial infarctions is atherosclerotic plaque disruption, which leads to coronary vessel obstruction, ${ }^{15}$ as well as prolonged ischemia. ${ }^{16}$ Perioperative myocardial ischemia is a consequence of an exaggerated sympathetic response, evidenced by increased myocardial oxygen demand and decreased coronary diastolic filling time (secondary to increases in heart rate and contractility caused by physiologic stress and elevated catecholamine levels). ${ }^{17}$

The exact mechanism by which beta-blockers reduce perioperative cardiac complications remains unclear. It has been proposed that through attenuation of the sympathetic response, with the major effects of reduction in heart rate (leading to increased perfusion time) and contractility (leading to decreased oxygen demand), perioperative beta-blockade results in a reduction in both cardiac caused mortality and the incidence of cardiovascular complications. ${ }^{18}$ Another hypothesis is that it is optimal heart rate control, rather than a specific agent, that is associated with a decline in ischemic events, as cardiac damage often results from inadequate myocardial blood flow. ${ }^{16}$ Additional potential cardiovascular effects of perioperative beta blockade include "vulnerable" coronary plaque stabilization and dysrhythmia prophylaxis. ${ }^{19}$

\section{Perioperative Beta-Blockade: Evolution of the Evidence}

Initial randomized trials, including the landmark study on which the original 1996 American College of Cardiology/American Heart Association (ACC/AHA) recommendations ${ }^{20}$ were based, suggested that beta-blockers reduce perioperative ischemia in patients with underlying arterial disease. ${ }^{18}$ Mangano et al ${ }^{18}$ performed a randomized, double-blind, placebo-controlled study comparing the effects of atenolol with those of a placebo on overall survival and cardiovascular morbidity in individuals undergoing noncardiac surgery with coronary artery disease (history of MI, typical angina, or atypical angina with positive stress test result) or risk factors for coronary artery disease $(\geq 2$ of the following: age $>65$ years, hypertension, current smoker, cholesterol level $>240 \mathrm{mg} / \mathrm{dL}$, or diabetes). Results of this study of 200 patients demonstrated that, in this population, perioperative atenolol (administered intravenously 30 minutes prior to surgery and continued by mouth throughout the hospital stay for up to 7 days) significantly reduced overall mortality and incidence of cardiovascular complications for as long as two years following discharge, with the primary effect identified as a decrease in mortality from cardiac causes during the first six to eight months. Atenolol administration, however, did not significantly reduce cardiac causes of mortality during hospitalization or incidence of perioperative MI. This lack of effect of atenolol in the perioperative period, however, may have been a result of the low incidence of significant perioperative cardiac events among study population patients, thus demonstrating a potential limitation of the study.

Subsequent research continued to contribute to a mounting body of evidence supporting perioperative beta-blockade in patients undergoing noncardiac surgery at high risk for adverse cardiac outcomes. In 1999, the Dutch Echocardiographic Cardiac Risk Evaluation Applying Stress Echocardiography (DECREASE) Study Group ${ }^{21}$ reported a randomized, non-blinded, placebocontrolled, multi-center trial examining the effect of bisoprolol on perioperative mortality and myocardial infarction in a subgroup of high-risk patients undergoing vascular surgery. High-risk patients were identified by the presence of both clinical risk factors and dobutamine stress echocardiography results positive for ischemia. Subjects were randomized and treatment group patients received standard perioperative care plus bisoprolol (initial dose at least one week prior to surgery and continued for thirty days postoperatively, with subsequent dosing individually adjusted by physician based on heart rate). Study endpoints were defined as cardiac death and myocardial infarction. A majority of the adverse cardiac events in both groups occurred during the first seven days following surgery. Overall study findings indicated a reduction in perioperative incidence of cardiac causes of mortality and nonfatal MI with the perioperative administration of bisoprolol. Despite the lack of significant clinical and echocardiogram differences between the standard care and treatment groups, the combined incidence of cardiac events in the standard care group was $34 \%$, compared to $3.4 \%$ in the bisoprolol group. This striking difference demonstrated a substantial benefit to treatment, 
significant enough to cause the investigation safety committee to suspend the study.

Based on investigation results, the DECREASE Study Group recommended that high-risk surgical patients receive beta-blockers perioperatively, with initiation one to two weeks preoperatively and continuation for at least two weeks postoperatively, with a goal of heart rate reduction to $<70$ beats/ minute preoperatively and $<80$ beats/minute in the immediate postoperative period. Investigators also suggested a potential alternative approach to standard preoperative management, proposing exclusion of preoperative non-invasive cardiac testing and replacement with prescription of perioperative beta-blockade for all patients with clinical risk factors undergoing high-risk surgery. Major limitations of this study were the fact that it was not blinded, thus allowing for potential bias and reporting error, and its focus only on a specific subpopulation. ${ }^{21}$

Prevention of perioperative morbidity and mortality is not the only consideration in the initial preoperative evaluation of a patient. Also important is the assurance of long-term future survival. As such, a related, follow-up study by the DECREASE Study Group ${ }^{22}$ assessed the long-term cardioprotective effects of perioperative and prolonged postoperative beta-adrenergic blockade with bisoprolol in the same, randomized cohort of high-risk patients following successful major noncardiac vascular surgery. Again, study treatment dosage was adjusted by physicians based on prescribed heart rate guidelines. Two-year follow-up was complete in all patients, monitoring for the occurrence of late cardiac events, including cardiac death and myocardial infarction. With no significant differences in clinical characteristic between the treatment and control groups, combined perioperative and long-term postoperative bisoprolol administration resulted in a significant, three-fold decrease in the incidence of late cardiac death and MI among high-risk patients after surviving major vascular surgery. However, similar to the previous study, patients and physicians were not blinded to the treatment. In addition, pre-trial risk of cardiac events in the two study groups may not have been comparable; the standard treatment group included survivors of perioperative MI, who may have consequently been at increased risk of late cardiac events during follow-up, and elevated incidence of perioperative mortality in the standard treatment group may also have eliminated the highest risk patients in this group.

Further trials evaluating the use of perioperative beta-blockers in patients undergoing noncardiac surgery did not produce favorable results, which challenges their efficacy and safety. Randomized controlled trials, including the Diabetic Postoperative Mortality and Morbidity (DIPOM) trial ${ }^{23}$ and the Metoprolol after Vascular Surgery(MaVS) study, ${ }^{24}$ demonstrated a lack of benefit of perioperative beta-blockade among specific patient populations, though there were limitations in the designs of these trials. More notably, the recent POISE trial, ${ }^{25}$ a large, multinational, multicenter, randomized, double-blind, placebo-controlled trial, was conducted to investigate the effects of perioperative beta-blockers in patients with risks for atherosclerosis or known atherosclerosis undergoing noncardiac surgery. Patients were randomized to receive either placebo or extended-release metoprolol succinate, which was initiated two to four hours prior to surgery and continued for 30 days postoperatively. Intention to treat analysis was conducted on a population of 8351 patients, and the primary endpoint was a composite of cardiovascular death, non-fatal myocardial infarction, and non-fatal cardiac arrest. At follow-up 30 days postoperatively, while significantly fewer treatment group patients reached the primary endpoint (an outcome driven entirely by a significant reduction in MI among treated patients), the incidences of both death and stroke were significantly greater in the metoprolol group. Though other factors may be responsible, investigators associated these findings primarily to the clinically significant hypotension and bradycardia associated with beta-blockade. Subsequent meta-analyses of trials of perioperative beta-blockers including POISE revealed some similarities in results and consistency with POISE findings. Meta-analyses demonstrated a risk reduction in non-fatal MI with beta-blockade, but also suggested an increased risk of death and non-fatal stroke with use of perioperative beta-blockers.

The POISE study represents the largest trial conducted to date addressing perioperative beta-blockers in patients undergoing noncardiac surgery. It is important to recognize, however, that this study too has its limitations. This trial utilized a very high and fixed dose of metoprolol (initial dose 2-8 times the commonly prescribed dose with no adjustment for heart rate). Additionally, therapy was initiated shortly (only hours) before surgery. Also of note is that in all of the meta-analyses conducted, POISE data accounted for approximately $80 \%$ of the patients. Results do, however, underscore the potential risks underlying the assumption that perioperative beta-blockade is beneficial and without harm.

\section{Evidence Limitations}

The role of beta-blockers in the perioperative setting is not yet clearly defined. Few randomized trials of medical therapy to prevent major perioperative adverse cardiac events have been performed, and most trials do not have sufficient power. Despite evidence from some trials demonstrating the potential benefit of perioperative beta-blockade in high-risk patients, much controversy still surrounds their use, especially given the methodological limitations of prior studies. No single agent, dose, route of administration, dosing schedule, or duration of treatment has been shown to be most effective. In addition, few studies have examined the effect of titration of therapy (i.e., to a target heart rate). Other controversial issues include the use of long- versus short-acting agents, effects of beta-blockade in patients on chronic beta-blocker therapy prior to surgery versus 
beta-blocker naive patients, and genetic variability in response to beta-blockade. ${ }^{26}$ Future research should aim to identify the optimal beta-blocking agent as well as define the role of perioperative beta-blockade in intermediate- and low-risk populations. To date, no literature has addressed the important topic of care-delivery mechanisms in the perioperative setting, identifying how, when, and by whom perioperative beta-blocker therapy should be implemented and monitored. More evidence is needed to corroborate the findings of previous studies and provide future direction. Further study will contribute to improved understanding, elucidation of these issues, and better clinical application of the available data.

\section{Current Guidelines}

While POISE trialinvestigators concluded aneed for revision of the approach to the use of perioperative beta-blockers, current ACC/ AHA guidelines, ${ }^{27}$ published in 2006, which include a focused update on perioperative beta-blocker therapy, still endorse a relatively wide indication for perioperative beta-blockade. Class I recommendations include: 1) continuation of beta-blockers in patients undergoing surgery who are prescribed beta-blocker therapy for angina, symptomatic arrhythmias, hypertension, or other ACC/AHA Class I guideline indications, and 2) administration of beta-blockers to patients undergoing vascular surgery at high cardiac risk with ischemia on preoperative testing. In addition, based on Class IIa recommendations, beta-blockers are probably recommended for 1) patients undergoing vascular surgery with evidence of coronary heart disease on preoperative testing, 2) patients in whom preoperative evaluation for vascular surgery identifies high cardiac risk, and 3) patients in whom preoperative assessment identifies coronary heart disease or high cardiac risk and who are undergoing intermediate- to high-risk procedures. According to Class IIb recommendations, beta-blockade may also be considered for 1) patients who are undergoing intermediate- or high-risk procedures in whom a single clinical cardiac risk factor is identified preoperatively, and 2) patients undergoing vascular surgery with low cardiac risk not currently on beta-blockers.

Though an approach to documenting cardiac risk different from the AHA/ACC guidelines, the Revised Cardiac Risk Index $(\mathrm{RCRI})^{7}$ is another extensively tested and accurate estimate of risk that can be utilized to guide care. ${ }^{28}$ The RCRI accounts for six variables: 1) high risk type of surgery, 2) ischemic heart disease, 3) history of congestive heart failure, 4) history of cerebrovascular disease, 5) insulin-dependent diabetes mellitus, and 6) chronic renal insufficiency (preoperative serum creatinine $>2.0 \mathrm{mg} / \mathrm{dL}$ ). Based on this method of preoperative risk stratification, subsequent risk of major cardiac complications with noncardiac surgery is estimated as illustrated in Figure 1, with and without beta-blocker therapy. ${ }^{28}$

\begin{tabular}{|l|c|c|c|}
\hline Figure 1. \\
\hline $\begin{array}{l}\text { Risk } \\
\text { Stratification }\end{array}$ & $\begin{array}{c}\text { RCRI } \\
\text { criteria }\end{array}$ & $\begin{array}{c}\text { Risk without } \\
\text { adrenergic } \\
\text { blockade (\%) }\end{array}$ & $\begin{array}{c}\text { Risk with } \\
\text { beta-blockade } \\
\text { (\%) }\end{array}$ \\
\hline Low Risk & 0 & $0.4-1.0$ & $<1$ \\
\hline $\begin{array}{l}\text { Intermediate } \\
\text { Risk }\end{array}$ & $1-2$ & $2.2-6.6$ & $0.8-1.6$ \\
\hline High Risk & $\geq 3$ & $>9$ & $>3$ \\
\hline
\end{tabular}

\section{Where Do We Go From Here?}

What, then, are the consequences of the results of the POISE trial for the use of perioperative beta-blockers in clinical practice? In using the treatment regimen outlined by the DECREASE Study Group, a low-dose, long-acting agent titrated to effect at least 7 days prior to surgery, a greater overall benefit to perioperative beta-blockade is seen as compared to risk. The POISE trial, however, demonstrates that acute administration of high-dose beta-blocker therapy may be associated with greater risk than benefit. Given all of the data, we do not believe that the evidence supports the initiation of prophylactic perioperative beta-blocker therapy in low- to intermediate risk patients (RCRI $£ 2$ ) undergoing noncardiac surgery. While beta-blockers do appear to decrease the risk of perioperative MI, the observed increases in the rates of stroke and mortality likely outweigh this benefit. A majority of the myocardial infarctions will be asymptomatic, but a few will result in serious complications in the perioperative period. In contrast, most strokes will be seriously disabling or incapacitating. However, perioperative beta-blockade still likely has significant cardioprotective effects in high-risk patients (RCRI $\geq 3$ ) or patients with evidence of significant ischemia by preoperative evaluation. In these patients, use of a beta-blocker perioperatively, with possible continuation in the postoperative period seems most prudent, with additional consideration for whether the patient is on chronic beta-blocker therapy prior to surgery or is beta-blocker naïve. Beta-blockers should be started orally days, if possible even weeks prior to the scheduled date of surgery to allow gradual up-titration of the dosage to achieve optimal heart rate and blood pressure control.

\section{Conclusion}

Cardiac complications of noncardiac surgery result in substantial morbidity and mortality, posing a serious health problem, whose magnitude only threatens to increase as the incidence of CAD rises and the population ages. As such, an accurate tool for cardiac risk assessment as well as sound guidelines for the subsequent prevention of such adverse outcomes is essential in moving forward. In select patients, perioperative beta-blockade may provide a safe and effective method of cardioprotection. However, it is crucial to highlight that as per the POISE trial, initiating perioperative beta-blockade is not without its own set of complications. Therefore, identification of the appropriate target population, avoidance of the adverse outcomes of such drugs, and future research aimed at characterizing the 
appropriate therapeutic regimen are needed in order to achieve the goal of reduced morbidity and mortality.

\section{References}

1. Heron MP, Hoyert DL, Murphy SL, Xu JQ, Kochanek KD, Tejada-Vera B Deaths: Final data for 2006. National Vital Statistics Reports, Vol. 57, No. 14. Hyattsville, MD: National Center for Health Statistics. 2009.

2. Gaziano Thomas A, Gaziano J. M, "Chapter 218. Epidemiology of Cardiovascular Disease” (Chapter). Fauci AS, Braunwald E, Kasper DL, Hauser SL, Longo DL, Jameson JL, Loscalzo J: Harrison's Principles of Internal Medicine, 17e: http://.accessmedicine.com.proxy1.lib.tju.edu:2048/content.aspx?aID=2871803.

3. Schouten O, Bax JJ, Poldermans D. Assessment of cardiac risk before noncardiac general surgery. Heart. 2006 Dec;92(12):1866-72.

4. Mangano D. Perioperative cardiovascular morbidity: new developments. Bailliere's Clin Anaesthesiol 1999;13:335-48.

5. Mangano DT, Goldman L. Preoperative assessment of patients with known or suspected coronary disease. N Engl J Med 1995;333(26):1750-6.

6. Devereaux PJ, Goldman L, Cook DJ, Gilbert K, Leslie K, Guyatt GH. Perioperative cardiac events in patients undergoing noncardiac surgery: A review of the magnitude of the problem, the pathophysiology of the events and methods to estimate and communicate risk. CMAJ. 2005;173:627-34.

7. Lee TH, Marcantonio ER, Mangione CM, Thomas EJ, Polanczyk CA, Cook EF, et al. Derivation and prospective validation of a simple index for prediction of cardiac risk of major noncardiac surgery. Circulation 1999;100:1043-9.

8. Ashton CM, Petersen NJ, Wray NP, Kiefe CI, Dunn JK, Wu L, et al. The incidence of perioperative myocardial infarction in men undergoing noncardiac surgery. Ann Intern Med 1993;118:504-10.

9. Mangano DT, Browner WS, Hollenberg M, London MJ, Tubau JF, Tateo IM. Association of perioperative myocardial ischemia with cardiac morbidity and mortality in men undergoing noncardiac surgery. The Study of Perioperative Ischemia Research Group. N Engl J Med 1990;323:1781-8.

10. Kumar R, McKinney WP, Raj G, Heudebert GR, Heller HJ, Koetting M, et al. Adverse cardiac events after surgery: assessing risk in a veteran population. J Gen Intern Med 2001;16:507-18

11. Detsky AS, Abrams HB, McLaughlin JR, Drucker DJ, Sasson Z, Johnston N, et al. Predicting cardiac complications in patients undergoing noncardiac surgery. J Gen Intern Med 1986;1:211-9.

12. Badner NH, Knill RL, Brown JE, Novick TV, Gelb AW. Myocardial infarction after noncardiac surgery. Anesthesiology 1998;88:572-8.

13. Shah KB, Kleinman BS, Rao TL, Jacobs HK, Mestan K, Schaafsma M. Angina and other risk factors in patients with cardiac diseases undergoing noncardiac operations. Anesth Analg 1990;70:240-7

14. Mangano DT. Perioperative cardiac morbidity. Anesthesiology 1990;72:153-84.

15. Dawood MM, Gutpa DK, Southern J, et al. Pathology of fatal perioperative myocardial infarction: implications regarding pathophysiology and prevention. Int J Cardiol 1996;57(1):37-44.
16. Raby KE, Brull SJ, Timimi F, et al. The effect of heart rate control on myocardial ischemia among high-risk patients after vascular surgery. Anesth Analg 1999;88(3):477-82

17. Maggio PM, Taheri PA. Perioperative Issues: Myocardial Ischemia and Protection - Beta-Blockade. Surgical Clinics of North America 2005;85:1091-1102.

18. Mangano DT, Layug EL, Wallace A, et al. Effect of atenolol on mortality and cardiovascular morbidity after noncardiac surgery. Multicenter study of Perioperative Ischemia Research Group. N Engl J Med 1996;335(23):1713-20.

19. London MJ, Zaugg M, Schaub MC, Spahn DR. Perioperative beta-adrenergic receptor blockade. Anesthesiology. 2004;100:170-175.

20. Eagle KA, Brundage BH, Chaitman BR, et al. Guidelines for perioperative cardiovascular evaluation for noncardiac surgery: report of the American College of Cardiology/American Heart Association Task Force on Practice Guidelines (Committee on Perioperative Cardiovascular Evaluation for Noncardiac Surgery). J Am Coll Cardiol. 1996;27(4):910-948.

21. Poldermans D, Boersma E, BaxJJ, et al. The effect of bisoprolol on perioperative mortality and myocardial infarction in high-risk patients undergoing vascular surgery. N Engl J Med. 1999;34:1789-1794.

22. Poldermans D, Boersma E, BaxJJ, et al. Bisoprolol reduces cardiac death and myocardial infarction in high-risk patients as long as two years after successful major vascular surgery. European Heart Journal 2001;22:1353-1358.

23. Juul AB, et al. The Diabetic Postoperative Mortality and Morbidity (DIPOM) trial: rationale and design of a multicenter, randomized, placebo-controlled, clinical trial of metoprolol for patients with diabetes mellitus who are undergoing major noncardiac surgery. Am Heart J. 2004;147(4):677-83.

24. Yang $\mathrm{H}$, et al. The effects of perioperative beta-blockade: results of the Metoprolol after Vascular Surgery (MaVS) study, a randomized controlled trial. Am Heart J. 2006;152(5):983-90.

25. POISE Study Group, Devereaux PJ, et al. Effects of extended-release metoprolo succinate in patients undergoing noncardiac surgery (POISE trial): a randomized controlled trial. Lancet 2008;371(9627):1839-47.

26. Domanski D, Schwarz ER. Is the Perioperative Use of Beta-Blockers Still Recommended? A Critical Review of Recent Controversies. J Cardiovasc Pharmacol Ther. 2009 [E-pub ahead of print].

27. Fleisher LA, Beckman JA, Brown KA, et al. ACC/AHA 2006 guideline update on perioperative cardiovascular evaluation for noncardiac surgery: focused update on perioperative beta-blocker therapy: a report of the american college of cardiology/american heart association task force on practice guidelines (writing committee to update the 2002 guidelines on perioperative cardiovascular evaluation for noncardiac surgery). J Am Coll Cardiol. 2006;47:2343-55.

28. Auerbach A, Goldman L. Assessing and reducing the cardiac risk of noncardiac surgery. Circulation. 2006;113:1361-76. 